\title{
DNA methylation level of the gene encoding thioredoxin-interacting protein in peripheral blood cells is associated with metabolic syndrome in the Japanese general population
}

\author{
Mirai Yamazaki ${ }^{1)}$, Hiroya Yamada ${ }^{2)}$, Eiji Munetsuna ${ }^{3)}$, Keisuke Maeda $^{4)}$, Yoshitaka Ando ${ }^{5)}$, \\ Genki Mizuno $^{6}$, Ryosuke Fujiii ${ }^{7)}$, Yoshiki Tsuboi ${ }^{7}$, Koji Ohashi ${ }^{5)}$, Hiroaki Ishikawa ${ }^{5}$, Shuji Hashimoto' ${ }^{2)}$, \\ Nobuyuki Hamajima ${ }^{8)}$ and Koji Suzuki ${ }^{7}$ \\ 1) Department of Medical Technology, Kagawa Prefectural University of Health Sciences, Takamatsu 761-0123, Japan \\ 2) Department of Hygiene, Fujita Health University School of Medicine, Toyoake 470-1192, Japan \\ 3) Department of Biochemistry, Fujita Health University School of Medicine, Toyoake 470-1192, Japan \\ 4) Department of Clinical Physiology, Fujita Health University School of Medical Sciences, Toyoake 470-1192, Japan \\ ${ }^{5)}$ Department of Biomedical and Analytical Sciences, Fujita Health University School of Medical Sciences, Toyoake 470-1192, Japan \\ 6) Department of Joint Research Laboratory of Clinical Medicine, Fujita Health University Hospital, Toyoake 470-1192, Japan \\ 7) Department of Preventive Medical Sciences, Fujita Health University School of Medical Sciences, Toyoake 470-1192, Japan \\ ${ }^{8)}$ Department of Healthcare Administration, Nagoya University Graduate School of Medicine, Nagoya 466-8550, Japan
}

\begin{abstract}
Metabolic syndrome (MetS) is cluster of metabolic diseases, including abdominal obesity, hyperglycemia, high blood pressure, and dyslipidemia, that directly escalate the risk of type 2 diabetes, heart disease, and stroke. Thioredoxininteracting protein (TXNIP) is a binding protein for thioredoxin, a molecule that is a key inhibitor of cellular oxidation, and thus regulates the cellular redox state. Epigenetic alteration of the TXNIP-encoding locus has been associated with components of MetS. In the present study, we sought to determine whether the level of TXNIP methylation in blood is associated with MetS in the general Japanese population. DNA was extracted from the peripheral blood cells of 37 subjects with and 392 subjects without MetS. The level of TXNIP methylation at cg19693031 was assessed by the bisulfitepyrosequencing method. We observed that TXNIP methylation levels were lower in MetS subjects (median $74.9 \%$, range $71.7-78.4 \%$ ) than in non-MetS subjects (median 77.7\%, range 74.4-80.5\%; $p=0.0024$ ). Calculation of the confounding factor-adjusted odds ratios (ORs) and 95\% confidence intervals (CIs) for hypomethylation revealed that subjects with MetS exhibited significantly higher ORs for hypomethylation than did those without MetS (OR, 2.92; 95\% CI, 1.33-6.62; $p=$ 0.009). Our findings indicated that lower levels of TXNIP methylation are associated with MetS in the general Japanese population. Altered levels of DNA methylation in TXNIP at cg19693031 might play an important role in the pathogenesis of MetS.
\end{abstract}

Key words: Metabolic disorder, Obesity, Thioredoxin-interacting protein (TXNIP), DNA methylation, Pyrosequencing

METABOLIC SYNDROME (MetS) has become a serious problem in developed countries, with an estimated prevalence of $25 \%$ in the world population [1]. MetS is a cluster of conditions that includes abdominal obesity, elevated glucose levels, high blood pressure, elevated triglycerides, and reduced levels of high-density

Submitted Jun. 4, 2021; Accepted Sep. 23, 2021 as EJ21-0339 Released online in J-STAGE as advance publication Oct. 14, 2021 Correspondence to: Koji Suzuki, Department of Preventive Medical Sciences, Fujita Health University School of Medical Sciences, 1-98 Dengakugakubo, Kutsukake-cho, Toyoake 470-1192, Japan.

E-mail:ksuzuki@fujita-hu.ac.jp lipoproteins. It is well accepted that MetS is a highly prevalent condition reflecting the cumulative effect of a series of factors, and that MetS increases the risk of type 2 diabetes mellitus (T2DM) and cardiovascular disease [2]. Indeed, MetS is considered one of the largest causes of morbidity and mortality worldwide.

There has been a recent explosion of interest in the notion that chronic low-grade inflammation is associated closely with MetS [3]. In addition, MetS is thought to be promoted by oxidative stress reflecting an imbalance in the oxidant/antioxidant state of the cell, resulting in turn from the excessive production of reactive oxygen species (ROS) $[4,5]$. Thioredoxin-interacting protein (TXNIP) is 
a binding protein for thioredoxin, a key inhibitor of cellular oxidation, and thus regulates the cellular redox state, leading to oxidative stress $[6,7]$. In response to ROS accumulation and the development of oxidative stress, TXNIP also plays an important role in inflammatory responses $[8,9]$. Depending on the generation of oxidative stress, TXNIP can serve as an activator of the nucleotide-binding oligomerization domain-like receptor family, pyrin domain-containing 3 (NLRP3) inflammasome, thereby regulating inflammation [10]. Thus, the association between dysregulation of TXNIP and the development of MetS has been the focus of recent work.

DNA methylation is an epigenetic modification that results in the placement of a methyl group on cytosines at $\mathrm{CpG}$ sites of DNA; this modification has been shown to alter the regulation of gene expression [11, 12]. Recently, there is growing evidence of the association between TXNIP DNA methylation and metabolic dysfunction. A recent study examined methylation at cg19693031, which is located at base $145,441,552$ (hg19) of chromosome 1, within a DNA sequence corresponding to the 3'-untranslated region (3'-UTR) of the TXNIP mRNA. Particularly in white blood cells, decreased methylation at $\operatorname{cg} 19693031$ has been shown to be closely associated with the levels of fasting blood glucose, glycated hemoglobin A1C (HbA1c), homeostasis model assessment of insulin resistance (HOMA-IR) values, and triglycerides, and the prevalence of T2DM [13-17]. Depending on the decreased methylation level at cg19693031, the levels of TXNIP mRNA in white blood cells are increased, which may result in the induction of inflammation [18]. TXNIP methylation in white blood cells may play a critical role in the pathogenesis of metabolic dysfunction, given that white blood cells themselves are responsible for oxidative stress and inflammation. Notably, Nuotio et al. recently reported that TXNIP methylation at $\operatorname{cg} 19693031$ in peripheral white blood cells is associated with MetS and its components [19]. Thus, methylation at $\operatorname{cg} 19693031$ in the TXNIP gene appears to be a candidate contributor to the pathogenesis of MetS.

Lifestyle includes different factors such as nutrition, behavior, stress, physical activity, working habits, smoking, and alcohol consumption. Increasing evidence shows that these lifestyle factors may influence epigenetic mechanisms, such as DNA methylation [20, 21]. Differences in culture, climate, and geography mean that lifestyle habits vary greatly between people of various regions and ethnicities. People in the United States and Canada, for example, consume more than $30 \%$ of their total calories from fat $[22,23]$. In contrast, people in Asia, such as the Japanese and Chinese, consume far less than $30 \%$ of their total energy intake in fat $[24,25]$. The distinct lifestyles of Western and Asian populations suggest the need to clarify the relationship between MetS and DNA methylation in various regional and ethnic groups. However, the relationship between MetS and TXNIP methylation has been reported (to our knowledge) only in a study examining European-derived populations [19].

Therefore, in this study, we investigated whether differential TXNIP methylation in peripheral blood cells was present in MetS compared to control subjects in the Japanese general population.

\section{Materials and Methods}

\section{Study subjects}

Since 1982, annual public health check-ups have been conducted for residents of Yakumo-cho, Hokkaido, Japan, aged 39 years or older. Various cross-sectional and longitudinal investigations of lifestyle-related diseases have enrolled individuals who participated in these health examinations [26-29]. We have collected epidemiological data at these annual health checkups, and have used these data as the basis of the Yakumo Study. The present cross-sectional study was undertaken in 525 individuals who participated in health examinations in 2015 as part of the Yakumo study. We excluded 96 subjects who had a clinical history of cancer or did not provide informed consent for the current study, or for whom the obtained blood samples were not adequate for the measurement of DNA methylation levels. The remaining 429 eligible individuals (189 men and 240 women) were included in the analysis described in the current report. The protocol for this study was approved by the Ethics Committee of Fujita Health University (Approval No. HG19-069).

\section{Collection of participant data}

Health information for each participant, including smoking habits (current, ever, or never), alcohol consumption (current, ever, or never), and a history of major illnesses (yes/no) were obtained by a trained public health nurse using self-administered questionnaires during the health examination. Height, weight, waist circumference, and blood pressure were measured during the health examination. The body mass index (BMI) was calculated by dividing weight $(\mathrm{kg})$ by height squared $\left(\mathrm{m}^{2}\right)$. Serum parameters were analyzed using an auto analyzer in the Yakumo Town Hospital laboratory.

\section{Assessment of metabolic syndrome}

The individuals diagnosed with MetS in the current study had central obesity, as defined by waist circumference ( $\geq 85 \mathrm{~cm}$ in men and $\geq 90 \mathrm{~cm}$ in women) plus at least 
two of the following three factors that define MetS; (1) elevated triglycerides $(\geq 150 \mathrm{mg} / \mathrm{dL})$ or decreased highdensity lipoprotein cholesterol (HDL-C) $(<40 \mathrm{mg} / \mathrm{dL})$, (2) elevated blood pressure (systolic $\geq 130 \mathrm{mmHg}$ or diastolic $\geq 85 \mathrm{mmHg}$ ), or (3) elevated fasting glucose ( $\geq 110 \mathrm{mg} / \mathrm{dL}$ ) [30]. Individuals without central obesity were classified as MetS component 0, while those with central obesity were classified as MetS component 1 . MetS components 2 to 4 were defined by the number of factors of either dyslipidemia, hypertension, or hyperglycemia, in addition to central obesity.

\section{Measurement of TXNIP DNA methylation}

DNA methylation analysis was conducted as described previously [31, 32]. Briefly, peripheral blood obtained under fasting conditions was collected into tubes containing ethylenediaminetetraacetic acid (EDTA)-2Na, and the buffy coat was obtained by centrifugation of these samples. DNA extracted from the buffy coat was bisulfite converted using the EpiTect Fast DNA Bisulfite Kit (QIAGEN, Hilden, Germany). We performed PCR using bisulfite-treated DNA $(10 \mathrm{ng} / \mu \mathrm{L})$ with the TaKaRa EpiTaq HS (for bisulfate-treated DNA; Takara, Otsu, Japan). Using PCR products, quantitative CpG methylation analysis was carried out on a PyroMark Q24 Advanced (QIAGEN) system with PyroMark Q24 Advanced Reagents (QIAGEN) in accordance with the manufacturer's instructions. We selected the following PCR and sequencing primers, as described in the previous study [33]: TXNIP: forward: 5'-TGTTTGTTGGATG GGTTTAAAAATAATT-3', reverse: 5'-biotin-AAACCT CCAAAAAACCTTAAAAAACTT-3', and sequencing: 5'-GGGTTAGgtAAAAATGG-3'. The results of $\mathrm{CpG}$ methylation level were analyzed using the PyroMark Q24 Advanced software (QIAGEN).

\section{Statistical analyses}

Statical analyses were performed using JMP v.14.0 (SAS Institute, Cary, NC, USA). The data for subject characteristics according to metabolic syndrome status are shown as mean \pm standard deviation (SD) or percentage. Analysis of differences between subjects with and without MetS were performed by two-tailed non-paired Student's $t$ tests. The DNA methylation levels of TXNIP were represented as geometric means and $25^{\text {th }}-75^{\text {th }}$ percentile ranges. The Cochran-Armitage test for trend was used to assess whether there was a monotonically decreasing trend in the TXNIP methylation level depending on the number of MetS components. We defined the hypomethylation group as the first tertile of the TXNIP DNA methylation levels (range 53.9\%-75.6\%). Multivariate adjusted odds ratios (ORs) and 95\% confidence intervals (CIs) for hypomethylation were calculated by logistic regression analysis according to the MetS and MetS components. We used age, sex, body mass index, smoking status, alcohol consumption, neutrophil percentage, and antihyperlipidemic or antihypertensive or antidiabetic medication use (user/non-user) as confounding factors. Probability values less than 0.05 were considered statistically significant.

\section{Results}

The characteristics of the subjects are presented in Table 1. A total of 37 cases with MetS (28 male, 9 female) and 392 subjects without MetS (161 male, 231 female) were enrolled in this study. No difference was observed in mean age between the two groups. By definition, subjects with MetS in this study had significantly higher BMI, waist circumference, triglycerides, systolic (SBP) and diastolic (DBP) blood pressure, and glucose,

Table 1 Subject characteristics according to metabolic syndrome status

\begin{tabular}{lccc}
\hline & $\begin{array}{c}\text { Non-MetS } \\
(N=392)\end{array}$ & $\begin{array}{c}\text { MetS } \\
(N=37)\end{array}$ & $p$-value \\
\hline Age (years) & $62.9 \pm 9.9$ & $61.7 \pm 10.4$ & 0.50 \\
\hline $\begin{array}{l}\text { Male/Female (\%) } \\
\text { Abdominal obesity }\end{array}$ & $41.1 / 58.9$ & $75.7 / 24.3$ & $<0.001$ \\
$\begin{array}{l}\text { BMI (kg/m²) } \\
\text { Waist circumference } \\
(\mathrm{cm})\end{array}$ & $23.2 \pm 3.2$ & $27.3 \pm 2.9$ & $<0.001$ \\
\hline
\end{tabular}

Dyslipidemia

Triglycerides $(\mathrm{mg} / \mathrm{dL})$

$98.0 \pm 57.4 \quad 205.9 \pm 123.1<0.001$

HDL cholesterol $(\mathrm{mg} / \mathrm{dL})$

$60.1 \pm 13.8 \quad 48.2 \pm 10.7 \quad<0.001$

$\begin{array}{lccc}\text { Hypertension } & & \\ \text { SBP }(\mathrm{mmHg}) & 130.0 \pm 20.7 & 144.2 \pm 15.4 & <0.001 \\ \text { DBP }(\mathrm{mmHg}) & 75.2 \pm 13.8 & 85.7 \pm 11.4 & <0.001\end{array}$

Hyperglycemia

Glucose $(\mathrm{mg} / \mathrm{dL}) \quad 88.3 \pm 14.2 \quad 102.6 \pm 25.7 \quad<0.001$

Metabolic components (\%)

$\begin{array}{lcc}0 & 74.2 & 0 \\ 1 & 7.7 & 0 \\ 2 & 18.1 & 0 \\ 3 & 0 & 91.9 \\ 4 & 0 & 8.1\end{array}$

Data are shown as mean \pm SD or as percentage.

Analysis of differences between groups performed by two-tailed Student's $t$ test.

Abbreviations: MetS, metabolic syndrome; BMI, body mass index; HDL, high density lipoprotein; SBP, systolic blood pressure; DBP, diastolic blood pressure. 
and significantly lower HDL-C (Table 1). Among subjects with MetS, 91.9\% had three of the MetS components, and $8.1 \%$ had four.

Methylation site $\operatorname{cg} 19693031$ in the TXNIP gene previously has been shown to be associated with various metabolic dysfunctions such as T2DM. Therefore, we analyzed the methylation level of cg19693031 using the bisulfite-pyrosequencing method. We observed that the methylation level at $\operatorname{cg} 19693031$ was lower in leukocyte samples from subjects with MetS (median $74.9 \%$, range 71.7-78.4\%) compared to subjects without MetS (median 77.7\%, range 74.4-80.5\%; $p<0.01$ ) (Fig. 1A). As shown in Fig 1B, a trend in the measured TXNIP methylation level was observed as the number of MetS components increased, although the difference fell short of statistical significance ( $p$ for trend $=0.08$ ).

To explore these results further, a logistic regression analysis was employed to verify the ability of TXNIP methylation level to discriminate MetS from non-MetS. The confounding factor-adjusted ORs and 95\% CIs for hypomethylation were calculated, and significantly higher ORs for hypomethylation were observed in subjects with MetS than in subjects without MetS (Table 2). For MetS components, there was a pattern of increasing ORs from one toward three or four MetS components, and subjects with three or four MetS components had statistically significant higher ORs for hypomethylation than did those with no MetS components (Table 3). As a next step, we divided the subjects by the type of MetS components, and calculated the confounding factoradjusted ORs and 95\% CIs for hypomethylation. We observed no significant difference in ORs between the subjects with and without abdominal obesity or hypertension. For dyslipidemia and hyperglycemia, significantly higher ORs for hypomethylation were observed in the subjects with either of these components compared to those lacking these components (Table 4).

\section{Discussion}

In the present study, we explored the link between MetS and TXNIP DNA methylation levels in white blood cells of the general Japanese population. We found a significant decrease in TXNIP DNA methylation level in our subjects with MetS compared to those without. Interestingly, a significant trend in the measured TXNIP DNA methylation level was observed with an increase in the number of MetS components: the DNA methylation was found to be lower in subjects with worse metabolic profiles. In addition, subjects situated in the tertiles with lower TXNIP methylation levels exhibited significantly greater risk of being affected by MetS when adjusted for sex, age, smoking habits, alcohol consumption, BMI, medication use, and percentage of neutrophils. Based on these results, we suggest that decreased levels of DNA methylation at cg19693031 in leukocytes is related to MetS in the general population of Japan.

The chronic low-grade inflammatory state associated
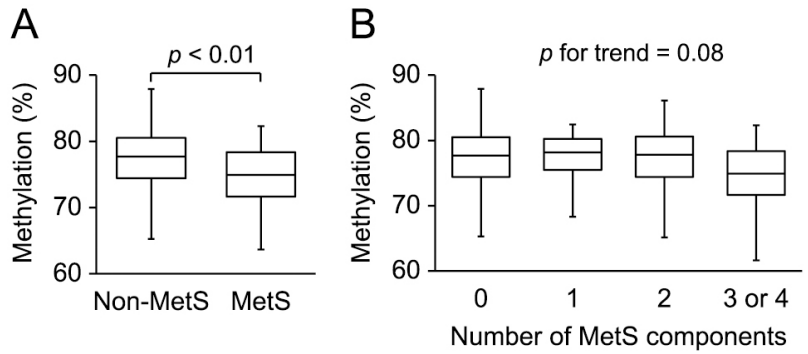

Fig. 1 DNA methylation levels of TXNIP in peripheral blood from subjects with and without MetS. (A) Boxplot showing distribution of methylation levels at $\operatorname{cg} 19693031$ $(T X N I P)$ in non-MetS and MetS subjects, as measured by pyrosequencing. The horizontal middle lines show the medians of the data, while the boxes show the 25 th to 75 th percentiles (Q1 and Q3). The whiskers represent maxima and minima. Two-tailed Student's $t$ tests were performed to test for differences between the two groups. (B) Comparison of TXNIP methylation level among subjects ranked by the number of MetS components is shown by a boxplot. The Cochran-Armitage test for trend was performed to assess whether there was a monotonically decreasing trend in the TXNIP methylation level depending on the number of MetS components.
Table 2 Multivariate adjusted ORs and 95\% CIs for TXNIP DNA hypomethylation according to MetS

\begin{tabular}{lccc}
\hline & $\begin{array}{c}\text { Hypomethylation } \\
(\%)\end{array}$ & $\begin{array}{c}\text { Adjusted ORs } \\
(95 \% \mathrm{CI})\end{array}$ & $p$-value \\
\hline Non-MetS & $122(31.1 \%)$ & Reference & \\
MetS & $22(59.5 \%)$ & $2.92(1.33-6.62)$ & $<0.01$ \\
\hline
\end{tabular}

Adjusted for age, sex, body mass index, smoking status, alcohol consumption, neutrophil percentage, and medication use

Table 3 Multivariate adjusted ORs and 95\% CIs for TXNIP DNA hypomethylation according to the number of MetS components

\begin{tabular}{lccc}
\hline $\begin{array}{c}\text { MetS } \\
\text { components }\end{array}$ & $\begin{array}{c}\text { Hypomethylation } \\
(\%)\end{array}$ & $\begin{array}{c}\text { Adjusted ORs } \\
(95 \% \mathrm{CI})\end{array}$ & $p$-value \\
\hline 0 & $91(31.3 \%)$ & Reference & \\
1 & $7(23.3 \%)$ & $0.41(0.15-1.13)$ & 0.08 \\
2 & $24(33.8 \%)$ & $0.93(0.44-1.96)$ & 0.85 \\
3 or 4 & $22(59.5 \%)$ & $2.47(1.00-6.11)$ & $<0.05$ \\
\hline
\end{tabular}

Adjusted for age, sex, body mass index, smoking status, alcohol consumption, neutrophil percentage, and medication use. 
Table 4 Multivariate adjusted ORs and 95\% CIs for TXNIP DNA hypomethylation according to the type of MetS components

\begin{tabular}{lccc}
\hline $\begin{array}{c}\text { MetS } \\
\text { components }\end{array}$ & $\begin{array}{c}\text { Hypomethylation } \\
(\%)\end{array}$ & $\begin{array}{c}\text { Adjusted } \\
\text { ORs } \\
(95 \% \mathrm{CI})\end{array}$ & $p$-value \\
\hline $\begin{array}{c}\text { Abdominal obesity } \\
\text { No }\end{array}$ & $91(31.3 \%)$ & $\begin{array}{c}\text { Reference } \\
0.92\end{array}$ & \\
Yes & $53(38.4 \%)$ & $\begin{array}{c}0.49-1.71) \\
(0.79\end{array}$ & \\
Dyslipidemia & $105(30.1 \%)$ & Reference & \\
No & $39(48.8 \%)$ & $\begin{array}{c}1.90 \\
(1.10-3.30)\end{array}$ & $<0.05$ \\
Yes & & & \\
Hypertension & $65(31.9 \%)$ & Reference & \\
No & $79(35.1 \%)$ & $\begin{array}{c}1.15 \\
(0.74-1.81)\end{array}$ & 0.53 \\
Yes & & & \\
Hyperglycemia & $125(31.7 \%)$ & Reference & \\
No & $19(54.3 \%)$ & 2.47 & $<0.05$ \\
Yes & & $(1.12-5.58)$ & \\
\hline
\end{tabular}

Adjusted for age, sex, body mass index, smoking status, alcohol consumption, neutrophil percentage, and medication use

with MetS has been implicated as a major factor in the establishment of MetS [34, 35]. However, the causes of the inflammatory conditions associated with MetS are not fully understood. TXNIP is an important redox regulator and plays an important role in inflammatory processes such as the activation of NLRP3 and the secretion of interleukin (IL)-1 $\beta[10,36]$. The up-regulation of TXNIP expression, which is influenced by the demethylation of cg19693031, orients peripheral blood cells toward an inflammatory activated state [18]. In addition, TXNIP-mediated inflammation is closely associated with the hyperglycemic state and lipid accumulation, which are risk factors for MetS [8, 36]. Thus, inflammatory pathways resulting from TXNIP methylation also may be involved in the pathogenesis of MetS. In fact, our data indicated that the level of $\operatorname{cg} 19693031$ methylation is decreased in subjects with MetS compared to those without MetS, a result that is consistent with the inference that TXNIP methylation is a strong candidate marker for MetS pathogenesis [19].

Our data indicated that TXNIP hypomethylation is associated with dyslipidemia and hyperglycemia among the MetS components. The methylation site $\operatorname{cg} 19693031$ in the gene TXNIP has been reported to be associated with lipid and glucose metabolism [37, 38], suggesting that these abnormalities might be the cause of TXNIP hypomethylation. However, logistic regression analysis showed that the methylation level of TXNIP was clearly different between MetS and non-MetS. Importantly, there was no significant difference in the OR for TXNIP hypomethylation between subjects with one or two MetS components and those without MetS components. The appearance of TXNIP hypomethylation is clearly distinguishable between MetS and non-MetS patients, suggesting that changes in TXNIP methylation levels might be caused by the development of MetS.

Nuotio et al. reported an association between the methylation of cg19693031 and MetS in Europeanderived populations [19]. However, for several reasons, that evidence was expected to have only limited application for the prediction of MetS in other geographic/ethnic groups. First, the diagnostic criteria for MetS differ among various countries [39]. For example, the waist circumference threshold for men in Japan is $\geq 85 \mathrm{~cm}$, while that in the USA and Canada is $\geq 102 \mathrm{~cm}$. Second, global leukocyte DNA methylation differs by geography and ethnicity, and these variables need to be taken into consideration in studies of global DNA methylation as an epigenetic marker for disease [40]. Finally, the fact that dietary habits, which significantly influence both the development of MetS and DNA methylation, differ among regions suggests the critical importance of validating the association between $\operatorname{cg} 19693031$ and MetS in non-European populations. In the present study, we found an association between DNA methylation of TXNIP and MetS, in agreement with the results reported by Nuotio et al. [19], despite the use of a distinct (Japanese) population compared to that (Europeans) used in the previous study. Our work also differed from the previous study in that we employed a pyrosequencing method. Pyrosequencing can detect and quantify even small changes in methylation level at selected $\mathrm{CpG}$ sites in specific target genes, and has been established as the "gold standard" for DNA methylation analysis [41]. Our findings indicated that $\operatorname{cg} 19693031$ in TXNIP may play an important role in the pathogenesis of MetS.

DNA methylation can be influenced by lifestyle factors such as diet, smoking, and stress. In fact, we recently reported that nutritional status has a significant impact on methylation patterns in blood [42-44]. We also have shown an association between DNA methylation level at cg19693031 and smoking behavior [33]. In this way, DNA methylation status in blood incorporates the influences of multiple lifestyle factors. Similarly, the relationship between MetS and long-term exposure to unsuitable habits also is recognized. Therefore, it seems possible that TXNIP hypomethylation occurs in response to adverse lifestyle habits, leading in turn to MetS.

DNA methylation has a number of advantages as a biomarker. First, DNA methylation patterns are robust, 
since this modification reflects the covalent attachment of methyl groups to DNA [45]. Second, DNA methylation analysis can be focused on specific and small regions of interest, given that aberrant DNA methylation occurs only in limited regions that encompass $\mathrm{CpG}$ sites. Third, as shown in the present study, analysis of methylation in DNA from peripheral blood samples is possible, enabling noninvasive testing. Taken together, these observations suggest that further technical advances and clinical studies may facilitate the successful implementation of epigenetic assays for the diagnosis of diseases, including MetS.

It is well established that MetS increases the incidence of T2DM. However, limited evidence is available regarding factors that may be predictive for the incidence of T2DM among subjects with MetS. In the present study, we showed that hypomethylation at $\operatorname{cg} 19693031$ is associated with MetS. Although epigenetic alterations at cg19693031 have been reported in subjects with T2DM $[15,16]$, most of our MetS subjects had not been diagnosed as overtly diabetic patients. Recently, growing evidence has suggested that epigenetic modifications, especially DNA methylation, may predispose humans to disease $[46,47]$. Based on these findings, we speculate that the level of methylation at $\operatorname{cg} 19693031$ may serve as a marker for T2DM risk assessment among subjects with MetS.

The frequency of MetS in elderly Japanese is expected to be approximately $20 \%[48,49]$. In this study, less than $10 \%$ of the subjects were classified as MetS. One of the reasons is that those taking medications for metabolic diseases were excluded from the MetS group. Since medication use has a significant impact on DNA methylation status [50, 51], those who use medications were not classified as MetS. It is necessary to note that our results are likely to be susceptibility to selection bias in that each metabolic abnormality may not be so severe.

The present study has several limitations. First, we did not examine the relationship between mRNA level and DNA methylation status of TXNIP in peripheral blood cells. Rong et al. reported the relationship between decreased methylation level of TXNIP and increased mRNA expression using a human monocytic cell line [18]. Furthermore, analyses using human peripheral blood leukocytes have shown a negative correlation between TXNIP methylation levels and TXNIP mRNA expression [52]. Therefore, TXNIP hypomethylation in the peripheral blood of subjects with MetS is predicted to result in increased TXNIP gene expression. Second, it is unclear whether the subtle methylation changes in the TXNIP gene observed in the present study induce the MetS phenotype. However, in recent years, it has become increasingly evident that small $(<10 \%)$ changes in DNA methylation levels are associated with complex disease phenotypes [53]. As subtle changes in methylation levels increasingly are being recognized as an epigenetic feature of complex disease phenotypes, changes in TXNIP methylation of even a few percent are expected to play an important role in the pathogenesis of MetS. Third, we did not analyze our subjects for any inflammatory biomarkers such as serum tumor necrosis factoralpha or C-reactive protein. Fourth, the current study analyzed only the relationship between MetS components and TXNIP hypomethylation. To further understand the role of TXNIP methylation on the pathology of MetS, TXNIP's relationship to insulin resistance needs to be analyzed. Fifth, the analysis of TXNIP DNA methylation was adjusted for the proportion of neutrophils. The methylation levels of peripheral blood cells are influenced by the differences resulting from varying proportions of white blood cell types [54]. Therefore, it remains possible that changes in TXNIP methylation rates in peripheral blood are influenced by cell composition. Finally, given that this work was a cross-sectional study, it is not clear whether the TXNIP hypomethylation observed in subjects with MetS is a cause or consequence of the development of MetS. DNA methylation abnormalities have been suggested to predispose to a variety of diseases. Moreover, DNA methylation is highly influenced by environmental factors, particularly lifestyle. These results lead us to speculate that hypomethylation of TXNIP gene is a cause of MetS development. To address these limitations, future studies will be needed to elucidate the relationship between TXNIP hypomethylation and MetS in more detail.

In summary, this study revealed that lower TXNIP methylation levels in peripheral blood cells are associated with MetS in subjects from the general Japanese population. As a result, altered levels of DNA methylation at $\operatorname{cg} 19693031$ may play an important role in the pathogenesis of MetS. TXNIP hypomethylation may serve as a biomarker or therapeutic target for MetS, although additional research will be required to further strengthen these observations.

\section{Acknowledgments}

We thank the participants and staff of the Health Examination Program for Residents of Yakumo, Hokkaido, Japan. This work was supported by the Japan Society for the Promotion of Science (JSPS) under Grants-in-Aid for Scientific Research Nos. JP26293144, JP17K09139, JP16H06277, JP19K24172, and JP20K10515. 


\section{Disclosure}

None of the authors have any potential conflicts of interest associated with this research.

\section{References}

1. O'Neill S, O'Driscoll L (2015) Metabolic syndrome: a closer look at the growing epidemic and its associated pathologies. Obes Rev 16: 1-12.

2. Eckel RH, Grundy SM, Zimmet PZ (2005) The metabolic syndrome. Lancet 365: 1415-1428.

3. Esposito K, Giugliano D (2004) The metabolic syndrome and inflammation: association or causation? Nutr Metab Cardiovasc Dis 14: 228-232.

4. Ilkun O, Boudina S (2013) Cardiac dysfunction and oxidative stress in the metabolic syndrome: an update on antioxidant therapies. Curr Pharm Des 19: 4806-4817.

5. Halliwell B, Cross CE (1994) Oxygen-derived species: their relation to human disease and environmental stress. Environ Health Perspect 102(Suppl 10): 5-12.

6. Shalev A (2014) Minireview: thioredoxin-interacting protein: regulation and function in the pancreatic $\beta$-cell. Mol Endocrinol 28: 1211-1220.

7. Chen J, Hui ST, Couto FM, Mungrue IN, Davis DB, et al. (2008) Thioredoxin-interacting protein deficiency induces Akt/Bcl-xL signaling and pancreatic beta-cell mass and protects against diabetes. FASEB J 22: 3581-3594.

8. Zhang X, Zhang JH, Chen XY, Hu QH, Wang MX, et al. (2015) Reactive oxygen species-induced TXNIP drives fructose-mediated hepatic inflammation and lipid accumulation through NLRP3 inflammasome activation. Antioxid Redox Signal 22: 848-870.

9. Devi TS, Lee I, Huttemann M, Kumar A, Nantwi KD, et al. (2012) TXNIP links innate host defense mechanisms to oxidative stress and inflammation in retinal Muller glia under chronic hyperglycemia: implications for diabetic retinopathy. Exp Diabetes Res 2012: 438238.

10. Zhou R, Tardivel A, Thorens B, Choi I, Tschopp J (2010) Thioredoxin-interacting protein links oxidative stress to inflammasome activation. Nat Immunol 11: 136-140.

11. Jones PA (2012) Functions of DNA methylation: islands, start sites, gene bodies and beyond. Nat Rev Genet 13: 484-492.

12. Yamada H, Munetsuna E, Yamazaki M, Mizuno G, Sadamoto N, et al. (2019) Maternal fructose-induced oxidative stress occurs via Tfam and Ucp5 epigenetic regulation in offspring hippocampi. FASEB J 33: 11431-11442.

13. Sayols-Baixeras S, Subirana I, Lluis-Ganella C, Civeira F, Roquer J, et al. (2016) Identification and validation of seven new loci showing differential DNA methylation related to serum lipid profile: an epigenome-wide approach. The REGICOR study. Hum Mol Genet 25: 4556-4565.

14. Dayeh T, Tuomi T, Almgren P, Perfilyev A, Jansson PA, et al. (2016) DNA methylation of loci within ABCG1 and PHOSPHO1 in blood DNA is associated with future type
2 diabetes risk. Epigenetics 11: 482-488.

15. Soriano-Tarraga C, Jimenez-Conde J, Giralt-Steinhauer E, Mola-Caminal M, Vivanco-Hidalgo RM, et al. (2016) Epigenome-wide association study identifies TXNIP gene associated with type 2 diabetes mellitus and sustained hyperglycemia. Hum Mol Genet 25: 609-619.

16. Florath I, Butterbach K, Heiss J, Bewerunge-Hudler M, Zhang Y, et al. (2016) Type 2 diabetes and leucocyte DNA methylation: an epigenome-wide association study in over 1,500 older adults. Diabetologia 59: 130-138.

17. Chambers JC, Loh M, Lehne B, Drong A, Kriebel J, et al. (2015) Epigenome-wide association of DNA methylation markers in peripheral blood from Indian Asians and Europeans with incident type 2 diabetes: a nested case-control study. Lancet Diabetes Endocrinol 3: 526-534.

18. Rong J, Xu X, Xiang Y, Yang G, Ming X, et al. (2020) Thioredoxin-interacting protein promotes activation and inflammation of monocytes with DNA demethylation in coronary artery disease. J Cell Mol Med 24: 3560-3571.

19. Nuotio ML, Pervjakova N, Joensuu A, Karhunen V, Hiekkalinna T, et al. (2020) An epigenome-wide association study of metabolic syndrome and its components. Sci Rep 10: 20567.

20. Lim U, Song MA (2012) Dietary and lifestyle factors of DNA methylation. Methods Mol Biol 863: 359-376.

21. Yamazaki M, Munetsuna E, Yamada H, Ando Y, Mizuno $\mathrm{G}$, et al. (2016) Fructose consumption induces hypomethylation of hepatic mitochondrial DNA in rats. Life Sci 149: 146-152.

22. Harcombe $Z$ (2019) US dietary guidelines: is saturated fat a nutrient of concern? Br J Sports Med 53: 1393-1396.

23. Kennedy ET, Bowman SA, Powell R (1999) Dietary-fat intake in the US population. J Am Coll Nutr 18: 207-212.

24. Elmadfa I, Kornsteiner M (2009) Dietary fat intake-a global perspective. Ann Nutr Metab 54(Suppl 1): 8-14.

25. Zhou BF, Stamler J, Dennis B, Moag-Stahlberg A, Okuda $\mathrm{N}$, et al. (2003) Nutrient intakes of middle-aged men and women in China, Japan, United Kingdom, and United States in the late 1990s: the INTERMAP study. J Hum Hypertens 17: 623-630.

26. Suzuki K, Yamada H, Fujii R, Munetsuna E, Ando Y, et al. (2021) Association between circulating vascularrelated microRNAs and an increase in blood pressure: a 5year longitudinal population-based study. J Hypertens 39: 84-89.

27. Tsuboi Y, Yamada H, Munetsuna E, Yamazaki M, Mizuno G, et al. (2018) Relationship between long interspersed nuclear element-1 DNA methylation in leukocytes and dyslipidemia in the Japanese general population. $J$ Atheroscler Thromb 25: 1231-1239. 
28. Munetsuna E, Yamada H, Ando Y, Yamazaki M, Tsuboi $\mathrm{Y}$, et al. (2018) Association of subcutaneous and visceral fat with circulating microRNAs in a middle-aged Japanese population. Ann Clin Biochem 55: 437-445.

29. Hida T, Imagama S, Ando K, Kobayashi K, Muramoto A, et al. (2018) Sarcopenia and physical function are associated with inflammation and arteriosclerosis in communitydwelling people: the Yakumo study. Mod Rheumatol 28: 345-350.

30. Alberti KG, Zimmet P, Shaw J, IDF Epidemiology Task Force Consensus Group (2005) The metabolic syndrome - a new worldwide definition. Lancet 366: 1059-1062.

31. Yamazaki M, Yamada H, Munetsuna E, Ishikawa $H$, Mizuno G, et al. (2018) Excess maternal fructose consumption impairs hippocampal function in offspring via epigenetic modification of BDNF promoter. FASEB $J 32$ : 2549-2562.

32. Mizuno G, Munetsuna E, Yamada H, Yamazaki M, Ando Y, et al. (2021) Maternal fructose consumption downregulates hippocampal catalase expression via DNA methylation in rat offspring. Nutr Res 92: 40-48.

33. Maeda K, Yamada H, Munetsuna E, Fujii R, Yamazaki M, et al. (2020) Association of smoking habits with TXNIP DNA methylation levels in leukocytes among general Japanese population. PLoS One 15: e0235486.

34. Sharma P (2011) Inflammation and the metabolic syndrome. Indian J Clin Biochem 26: 317-318.

35. de Ferranti S, Mozaffarian D (2008) The perfect storm: obesity, adipocyte dysfunction, and metabolic consequences. Clin Chem 54: 945-955.

36. Perrone L, Devi TS, Hosoya K, Terasaki T, Singh LP (2009) Thioredoxin interacting protein (TXNIP) induces inflammation through chromatin modification in retinal capillary endothelial cells under diabetic conditions. J Cell Physiol 221: 262-272.

37. Walaszczyk E, Luijten M, Spijkerman AMW, Bonder MJ, Lutgers HL, et al. (2018) DNA methylation markers associated with type 2 diabetes, fasting glucose and $\mathrm{HbAlc}$ levels: a systematic review and replication in a casecontrol sample of the Lifelines study. Diabetologia 61: 354-368.

38. Petersen AK, Zeilinger S, Kastenmuller G, RomischMargl W, Brugger M, et al. (2014) Epigenetics meets metabolomics: an epigenome-wide association study with blood serum metabolic traits. Hum Mol Genet 23: 534545.

39. Lear SA, Gasevic D (2019) Ethnicity and metabolic syndrome: implications for assessment, management and prevention. Nutrients 12: 15.

40. Zhang FF, Cardarelli R, Carroll J, Fulda KG, Kaur M, et al. (2011) Significant differences in global genomic DNA methylation by gender and race/ethnicity in peripheral blood. Epigenetics 6: 623-629.

41. Poulin M, Zhou JY, Yan L, Shioda T (2018) Pyrosequencing methylation analysis. Methods Mol Biol 1856: 283
296.

42. Fujii R, Yamada H, Munetsuna E, Yamazaki M, Mizuno $\mathrm{G}$, et al. (2021) Dietary fish and $\omega-3$ polyunsaturated fatty acids are associated with leukocyte ABCA1 DNA methylation levels. Nutrition 81: 110951.

43. Fujii R, Yamada H, Munetsuna E, Yamazaki M, Ando Y, et al. (2019) Associations between dietary vitamin intake, ABCA1 gene promoter DNA methylation, and lipid profiles in a Japanese population. Am J Clin Nutr 110: 12131219.

44. Fujii R, Yamada H, Munetsuna E, Yamazaki M, Mizuno $\mathrm{G}$, et al. (2019) Dietary vegetable intake is inversely associated with ATP-binding cassette protein A1 (ABCA1) DNA methylation levels among Japanese women. Nutrition 65: 1-5.

45. Talens RP, Boomsma DI, Tobi EW, Kremer D, Jukema JW, et al. (2010) Variation, patterns, and temporal stability of DNA methylation: considerations for epigenetic epidemiology. FASEB J 24: 3135-3144.

46. Nilsson E, Ling C (2017) DNA methylation links genetics, fetal environment, and an unhealthy lifestyle to the development of type 2 diabetes. Clin Epigenetics 9: 105.

47. Menon R, Conneely KN, Smith AK (2012) DNA methylation: an epigenetic risk factor in preterm birth. Reprod Sci 19: 6-13.

48. Aizawa Y, Kamimura N, Watanabe H, Aizawa Y, Makiyama Y, et al. (2006) Cardiovascular risk factors are really linked in the metabolic syndrome: this phenomenon suggests clustering rather than coincidence. Int $J$ Cardiol 109: 213-218.

49. Ishizaka N, Ishizaka $Y$, Toda E, Hashimoto H, Nagai R, et al. (2005) Hypertension is the most common component of metabolic syndrome and the greatest contributor to carotid arteriosclerosis in apparently healthy Japanese individuals. Hypertens Res 28: 27-34.

50. Gao X, Colicino E, Shen J, Just AC, Nwanaji-Enwerem JC, et al. (2018) Accelerated DNA methylation age and the use of antihypertensive medication among older adults. Aging (Albany NY) 10: 3210-3228.

51. Garcia-Calzon S, Perfilyev A, Mannisto V, de Mello VD, Nilsson E, et al. (2017) Diabetes medication associates with DNA methylation of metformin transporter genes in the human liver. Clin Epigenetics 9: 102.

52. Wang XB, Han YD, Zhang $\mathrm{S}$, Cui $\mathrm{NH}$, Liu ZJ, et al. (2016) Associations of polymorphisms in TXNIP and gene-environment interactions with the risk of coronary artery disease in a Chinese Han population. $\mathrm{J}$ Cell Mol Med 20: 2362-2373.

53. Leenen FA, Muller CP, Turner JD (2016) DNA methylation: conducting the orchestra from exposure to phenotype? Clin Epigenetics 8: 92.

54. Reinius LE, Acevedo N, Joerink M, Pershagen G, Dahlen SE, et al. (2012) Differential DNA methylation in purified human blood cells: implications for cell lineage and studies on disease susceptibility. PLoS One 7: e41361. 